\title{
DE L'HOMME ET DE DIEU DANS LA PHILOSOPHIE DE MAX SCHELER
}

Heinz Leonardy*

SINTESE - Este artigo procura apresentar a significativa evolução das relaçőes entre 0 Homem e o Absoluto no pensamento de Max Scheler. Num primeiro momento, o Homem deve estar contente com ser, como se fosse um Gottsucher, está em vias de ser, no posterior trabalho, o verdadeiro sócio para um "futuro Deus". A história do homem e a história de Deus tende a ser somente uma única história. PALAVRAS-CHAVE - Absoluto. Tornar-se. Deus. História. Homem. Scheler.

\begin{abstract}
This paper endeavours to present the significative evolution of the relationship between Man and the Absolute in Max Scheler's thought. Whereas, in a first approach, Man had to be content with being, as it were, a "Gottsucher", he is going to be, in Scheler's later work, the real fellow-worker for a "becoming God". The history of Man and the history of God turn to be only one History.

KEY WORDS - Absolute. Becoming. God. History. Man. Scheler.
\end{abstract}

En premier lieu nous tenons à vous remercier de nous avoir invité à ce Symposium organisé à l'occasion de la fondation de la société brésilienne de phénoménologie. Être appelé ainsi comme une sorte de témoin à une naissance sinon un baptême fait énormément plaisir et ce principalement pour la raison que vous fournissez une preuve évidente que ce mouvement, inauguré en l'année 1900 a encore de beaux jours devant lui. Ce n'est certes pas à nous de définir, de déterminer en termes précis l'à-venir de la phénoménologie, c'est à nous tous d'en esquisser les contours, les possibilités et les limites.

Ceci dit, les organisateurs, en l'occurrence le secrétaire exécutif, M. Nythamar de Oliveira, nous ont permis - ce dont nous les remercions aussi - de parler d'un auteur et d'un sujet qui ne rentrent pas "tel quel" dans le programme annoncé. Car c'est ni de Husserl ni de Heidegger que nous allons vous entretenir, mais d'un penseur qui se situe entre les deux. De plus le sujet que nous avons choisi nest pas non plus, au début du moins, une question "classique" de la phénoménologie puisque ni Husserl ni Heidegger n'y ont apporté une attention particulière. C'est peut-être le moment de revoir, de réexaminer la notion-même de "questions classiques de la phénomé-nologie". L'anthropologie (au sens philosophique conven-

\footnotetext{
Université de Louvain - Centre d'Études Phénoménologiques.
}

\begin{tabular}{|l|l|l|l|l|l|}
\hline VERTTAS & Porto Alegre & v. 45 & n. 1 & Março 2000 & p. 51-66 \\
\hline
\end{tabular}


tionnel), la métaphysique et le discours sur Dieu ne font-ils pas partie intégrante du questionnement phénoménologique?

L'auteur et le sujet ainsi annoncés, nous devons encore indiquer l'attitude à partir de laquelle nous allons les aborder. Ce n'est pas de l'extérieur, mais de l'intérieur que nous allons les approcher. Et en cela nous suivons parfaitement Max Scheler. Dans la relation d'une personne à une autre personne le Mit est le "concept opératoire" central. Et nous nous situons d'emblée dans cette dimension du Mit, du Mitdenken en l'occurrence. Mais, aux yeux de Scheler, ceci ne suffit pas encore à vraiment saisir l'autre: en effet, il faut en outre que ce vouloircomprendre soit fondé in fine dans l'amour. L'acte de compréhension doit être sous-tendu et guidé par la sympathie, une des sous-formes de l'amour.

Cette attitude nous permettra d'un côté de ne pas "objectiver" (au sens de chosifier ou simplement de figer) cette pensée - toute pensée philosophique doit rester une pensée ouverte - mais cette sympathie appelle d'un autre côté une réserve importante: il s'agira de ne pas se laisser séduire. Presque tous les auditeurs et les commentateurs de la première génération soulignent aussi bien la génialité que la séduction exercée par ce penseur. Si nous avons donc l'intention d'adopter l'attitude que nous avons dite, cela n'implique nullement que nous sommes toujours d'accord sur le contenu de sa pensée. À la fin nous aurons l'occasion de revenir sur ce point, puisque le sujet choisi est particulièrement délicat et illustre parfaitement ce "devoir de réserve" que nous venons d'évoquer.

Il serait prétentieux sinon vaniteux de vouloir étendre à chaque fois cet effort de compréhension à l'ensemble de la pensée d'un auteur. Et ceci est particulièrement vrai dans le cas qui nous préoccupe ici. La philosophie de Scheler comprend tant de facettes qu'il est tout simplement impossible de l'embrasser d'un seul regard. Et en outre, il faut tenir compte du fait qu'elle a connu une évolution importante (personnellement nous préférons de loin ce concept d'évolution à celui de rupture employé par beaucoup de commentateurs; mais encore une fois, le sujet choisi se prête parfaitement à ce que tout un chacun puisse se forger son opinion à ce sujet). Par conséquent nous nous contenterons d'essayer de lever un coin du voile qui recouvre cette pensée - à nos yeux un sens possible du dévoiler heideggerien, lequel signifie notamment que par ce dévoilement nous risquons de voiler d'autres facettes de sa philosophie - ou encore, pour adopter le langage husserlien, nous devons procéder par Abschattungen, ce que nous interprétons ici de la même manière: arracher à l'ombre; mais, et ceci est capital, nous tenterons de maintenir en arrière-fond une vision globale, une sorte de Gesamtschau qui permettrait de relier la partie au tout. Ceci nous semble d'autant plus important dans le cas de Max Scheler, car, il ne faut se voiler la face: Scheler est très peu connu de nos jours, il est tombé dans l'ombre sinon dans l'oubli. ${ }^{1}$

1 C'est pour cette raison qu'a été fondée en 1993 une "Max-Scheler-Gesellschaft" dont le but est justement de promouvoir et l'étude et la diffusion de sa philosophie. Par ailleurs on peut trouver une foule de renseignements sur Scheler et sur cette société sur le site créé par Manfred S. Frings sur le web à l'adresse: http://members.aol.com/fringsmk/Scheler.htm. 
Notre propos portera sur le volet de sa pensée dont l'évolution est la plus frappante: la relation de l'homme à Dieu qui est de toute évidence également celle de Dieu à l'homme. Dans une précédente publication ${ }^{2}$ nous avions repris, à la lumière de Scheler, l'expression de Nietzsche: "Ou'il est difficile d'être un homme". Cette fois-ci nous tenterons de jeter un regard plus large sur cette corrélation. Pour ce faire nous procéderons en deux étapes: en un premier temps nous examinerons cette "cohabitation" dans la période dite catholico-phénoménologique de sa philosophie (d'environ 1902 à 1920/22), ensuite cette "coopération" dans sa phase qualifiée souvent de métaphysico-panenthéiste (de 1920/22 à sa mort en 1928). ${ }^{3}$ Nous découvrirons ainsi ce qu'il a appelé lui-même "un développement considérable de sa pensée" (GW 2, 17). ${ }^{4}$

Au moment où la problématique de la relation de l'homme à Dieu fait son apparition dans la pensée schelerienne, tout semblait aller de soi et se dégageait même une odeur de "déjà vu". Alors que dans la phase dite néo-kantienne cette problématique n'était encore nullement thématisée, elle va très vite occuper une place importante lors de l'élaboration de sa phénoménologie propre et ce selon deux voies différentes: d'une part du côté de sa phénoménologie des valeurs où non pas encore Dieu, mais la déité apparaît comme porteuse de la modalité suprême (déjà) des valeurs, à savoir le sacré. D'autre part, au moment où sa phénoménologie se mue pour ainsi dire en personnalisme phénoménologique, Dieu est nommé presque automatiquement la "personne des personnes" (GW 2, 396), "la personne infinie purement et simplement (schlechthin)" (GW 2, 514), et ce n'est qu'en Dieu que nous pouvons saisir la valeur absolument suprême (GW 2, 47). Et ce Dieu par rapport à l'homme? "Dieu - c'est au plus haut degré la seule personne parfaite et pure. Et ce n'est qu'une 'personne' imparfaite, allégorique, ce qui parmi les hommes peut s'appeler ainsi" (GW 3, 190). S'installe ainsi une relation claire et limpide dont la transparence devient encore plus grande quand on examine la source de cette conception. D'ailleurs Scheler nous l'indique clairement lui-même quand il identifie cette relation à la relation filiale où l'homme se résume pour ainsi dire à assumer son rôle d'"enfant de Dieu". II n'y a par conséquent aucun doute que la source soit la doctrine chrétienne ou - à cause de ses attaques parfois virulentes contre le protestantisme - pour être plus précis: la doctrine catholique. Et la pensée schelerienne à ce sujet peut être considérée, doit

LEONARDY Heinz, “'Es ist schwer, ein Mensch zu sein'. Zur Anthropologie des späten Scheler”, in Studien zu Max Scheler (Phänomenologische Forschungen, Bd. 28/29), 1994, pp. 71-94.

Par souci de complétude mentionnons encore la première phase de son travail philosophique, la période dite néokantienne qui va de sa dissertation doctorale (1897) à sa rencontre avec Husserl en 1902.

Avant-propos (1926) à la troisième édition du Formalisme (dont la première édition date de 1913). Nos citations de Scheler sont tirées des Gesammelte Werke (GW), éditées d'abord par Maria Scheler et ensuite par Manfred S. Frings et publiées pour les premières par le Francke-Verlag (Bern et Munich), les dernières par le Bouvier-Verlag de Bonn. 
même être considérée comme un effort pour étayer philosophiquement, c'est-àdire en ce cas phénoménologiquement, cette doctrine non philosophique. L'essentiel de sa philosophie de la religion a été élaboré entre 1915 et 1920 et est consigné principalement dans deux contributions: "Problèmes de la religion" (GW $5,101-353$ ) et "Sphère absolue et position de la réalité 'de l'idée' de Dieu" (GW 10, 179-253). Ce n'est nullement notre intention de faire un exposé plus ou moins exhaustif de ce volet de sa philosophie, mais il nous semble néanmoins indispensable de décrire quelque peu la relation de l'homme à Dieu dans cette perspective - indispensable, si nous voulons comprendre ce que nous nous plaisons à nommer un "tournant" (Kehre) dans la pensée schelerienne et ce que d'aucuns appellent une rupture.

Et d'abord, quelle est la place, la situation de l'homme dans le Tout? Scheler nous dit que "l'erreur des doctrines existantes sur l'homme consiste en ce qu'on voulait intercaler entre la 'vie' et Dieu une étape immuable, quelque chose de définissable comme essence: l'homme'. Mais cette étape n'existe pas et l'indéfinissabilité appartient justement à l'essence de l'homme. Il n'est qu'un 'intervalle', une 'frontière', une 'passerelle', un 'apparaitre de Dieu' dans le flux de la vie et un éternel 'dépassement d'elle-même de la vie'" (GW 3, 186). Et notre auteur de nous indiquer tout de suite dans son essai "A propos de l'idée de l'homme" (GW 3, 171-195) vers quoi doit s'orienter ce dépassement: vers un quelque chose qui porte le nom de Dieu. L'homme en ce sens est l'être qui peut (tout le monde n'y arrive pas) transcender la vie et qui peut se transcender lui-même. I est l'intention et le geste de la transcendance elle-même, il est "le X vivant qui cherche Dieu!" (ibid.). Et il peut l'être dans la mesure où son entendement, ses outils et ses machines lui donnent de plus en plus de loisir pour contempler et aimer Dieu. Ce n'est que cela qui peut justifier son entendement et son œuvre, à savoir la civilisation: qu'ils rendent son être de plus en plus perméable pour l'esprit et l'amour dirigés vers ce quelque chose qui porte le nom de Dieu. Et Scheler de nous fournir une quasi-définition de l'homme: "Une chose qui commence à se dépasser elle-même et à chercher Dieu - c'est cela justement l'homme', quelle que soit sinon son apparence" (GW 3, 189). Et d'insister encore: L'homo bestia naturalis ne devient vraiment homo qu'au moment où le désir de Dieu mobilise tout être et tout esprit contre ce qui n'est que "monde". L'homme ne devient vraiment homme que quand il est fatigué d'être simplement un homme.

Par essence l'homme est donc transcendance ou, comme le dit encore Scheler: il est "le porteur d'une tendance, qui transcende toutes les valeurs vitales possibles et qui est orientée vers le 'divin', ou pour le dire de manière plus concise: il est le chercheur de Dieu (Gottsucher)" (GW 2, 296). Ce terme de "Gottsucher" lui a été plus que probablement inspiré par les fameux "Goldsucher", les chercheurs d'or, mais il est important de signaler une différence fondamentale entre les deux types de chercheurs: alors que le chercheur d'or part dans l'inconnu, le chercheur de Dieu est en quête de quelque chose qu'il a déjà en lui 
depuis toujours. Scheler se refère ici expressément à la parole de Pascal: "Tu ne me chercherais pas si tu ne m'avais déjà trouvé".

À l'homme est ainsi dévolue une tâche ou - ce terme nous semble plus adéquat dans ce contexte - une mission, à savoir la recherche de Dieu. Et Scheler d'affimer d'emblée que nous avons un certain accès à Dieu, mais puisqu'il s'agit d'une relation de personne à personne, c'est-à-dire d'un centre d'actes à un autre centre d'actes (cfr. la définition de l'essence de la personne dans GW 2, 382), cet accès devra se situer au niveau d'un acte. Dans son analyse des différents modes essentiels d'actes, modes irréductibles les uns aux autres, il place en tête, comme mode suprême donc, l'acte religieux. "La première vérité de toute phénoménologie de la religion ... est loriginalité et la non-déductibilité de l'expérience religieuse" (GW 5, 170). Comme p.ex. les valeurs ne nous sont accessibles que dans l'acte du sentir spirituel (les actes spirituels étant le mode essentiel d'actes juste en-dessous du mode supérieur), Dieu l'est exclusivement dans l'acte religieux. En procédant de la sorte, Scheler réclame d'emblée pour l'acte religieux la même évidence eidétique que pour tous les autres actes de la conscience. Sa philosophie de la religion se veut donc eo ipso une phénoménologie de la religion qui nous procure la saisie des essences. "Ces actes 'religieux' appartiennent dans leur genre essentiel à la conscience humaine de manière aussi constitutive que penser, juger, percevoir, se souvenir" (GW 5, 242).

Avant d'examiner d'un peu plus près ce que nous livre l'acte religieux ou l'expérience religieuse sur Dieu - ce deus absconditus, caché parce que dépassant infiniment et radicalement tout horizon humain (GW 3, 81) -, il nous faut insister sur le présupposé majeur de toute connaissance de Dieu comme de toute connaissance d'autrui d'ailleurs. Comme toute personne peut se refuser à notre approche, peut s'emmurer dans le silence, Dieu pourrait le faire également, il pourrait se taire (c'est le privilège de la seule personne de pouvoir faire silence). Mais, selon Scheler, son amour infini ne le lui permet pas - malgré sa toute-puissance (cfr. GW 10, 187). Et toute notre connaissance de Dieu est par conséquent basée sur ce souhait divin de se faire connaitre et son degré dépendra d'un côté de ce que Dieu veut bien nous communiquer de lui-même et de l'autre de notre capacité ou aptitude à saisir cette auto-communication divine. Notre accès à Dieu n'est donc possible que grâce à la révélation qui est auto-révélation, auto-communication (Selbstmitteilung) de Dieu.

Qu'en est-il alors de notre connaissance de la réalité divine, de l'existence réelle de Dieu? En premier lieu Scheler nous donne ce qu'il appelle le principe épistémologique fondamental d'une possible expérience d'un Dieu réel, principe qui rejoint ce qui précède: "Si quelque chose de l'essence du divin existe aussi réellement, alors il n'y a qu'une manière dont sa réalité peut être donnée ... à des personnes finies: qu'il se donne lui-même spontanément à connaître à ceux-ci ... Et si ce réel est une personne, ce se-donner-à-connaitre-comme-réel ne peut être que l'auto-révélation (Selbstoffenbarung) de cette personne" (GW 10, 185). Le "si" initial indique clairement que Scheler n'admet aucune preuve "philosophique" (et certainement pas une preuve "rationnelle"; si preuve il y avait, elle viendrait pour 
lui du champ axiologique auquel la connaissance théorique est aveugle) de l'existence de Dieu puisque à ses yeux la philosophie est "connaissance sans révélation", et en tant que telle "ne peut qu'exhiber le mode constitutif d'expérience, qui, conformément au rapport essentiel de tels modes déterminés d'être et de valeur avec des modes d'actes et des formes de connaissance, 'appartient' au domaine d'objets du divin, mais qui doit s'en remettre à une expérience contingente et factuelle de cet être-d'expérience déterminé a priori pour constater cette réalité dans un acte de croyance" (GW 10, 184). Mais, comme si Scheler voulait atténuer ce qui pourrait être ressenti comme une déficience propre à la seule connaissance de Dieu, il élargit cette déficience en disant que toute "conscience de la réalité" de quelque chose est quant à son mode de conscience toujours conscience de croyance ... et ceci vaut aussi bien pour la conscience de la réalité d'un Dieu que pour celle d'une table (GW 10, 243).

Ensuite: que pouvons-nous connaittre de Dieu? La même question sous l'autre angle: qu'est-ce que Dieu veut bien communiquer de lui-même? Qu'il se communique par auto-révélation, nous l'avons appris entretemps. Cette dernière peut s'effectuer selon deux modes. Soit par ce que Scheler appelle la révélation positive et qui est pour lui la forme la plus haute de l'autocommunication: comme le divin est un être de la forme de la personnalité, cette révélation est médiatisée par la parole et une ou des personnes. Il s'agit donc ici d'une personne qui se révèle à une personne via une personne ou d'autres personnes. Soit par la forme la plus "courante" que Scheler appelle la révélation naturelle où Dieu se présente luimême, s'expose en quelque sorte dans les choses, les événements de la réalité naturelle, psychique, sociale et historique. Ici la tâche de l'homme est de découvrir et de décrypter les traces que Dieu a laissées derrière lui dans la création, que ce soit dans la matière, dans la nature vivante, dans l'âme humaine, dans la société ou dans l'histoire. Autant de manières pour Dieu de se révéler à chaque fois autrement et de manière plus ou moins adéquate (cfr. GW 5, 157-8).

Dans l'attitude naturelle religieuse l'homme reçoit - Dieu dévoile - d'emblée deux déterminations essentielles du divin: il est étant absolu (Ens a se) et il est sacré. De cette conscience naturelle d'un étant absolu il s'ensuit presque automatiquement que l'homme s'insère dans la sphère de l'étant relatif et qu'il en déduit la totale dépendance de cette sphère par rapport à la sphère absolue, c'est-à-dire Dieu. Celui-ci, en tant qu'Ens a se, nous apparait d'emblée comme activité pure, force et pouvoirs purs alors que du côté de l'étant relatif n'apparaît que passivité, impuissance et dépendance. Nous sont ainsi révélés d'entrée de jeu la toutepuissance de Dieu ainsi que l'infériorité et l'impuissance, du moins partielle, de l'homme. Dans sa première saisie l'acte religieux nous livre par conséquent ces trois déterminations essentielles - élevées par Scheler au rang d'évidences eidétiques - du divin que sont l'absoluité, la toute-puissance et le sacré. En un deuxième mouvement nous est révélé que le monde (au sens le plus large) est et qu'il est l'œuvre de Dieu, et c'est en tant que création (le monde en tant qu'expression de la surabondance de l'amour divin qui nourrit cette création comme une source jaillissant sans cesse) qu'il est la révélation possible de son 
essence même. Du contenu essentiel du monde nous pouvons déduire, par analogie, les attributs que Scheler qualifie de supraformels - les trois premiers déjà cités étant des attributs formels - comme p.ex. esprit, raison, volonté, amour, omniscience, bonté infinie, créateur etc. Mais puisqu'ils sont tirés de notre expérience du monde, ces attributs ne peuvent être assignés à Dieu que de manière inadéquate, non explicite et sur le mode analogique - puisque Dieu est par principe, ou principiellement, transcendant par rapport à toutes les catégories de l'entendement humain et que par conséquent - ce que la métaphysique anthropomorphique n'a pas vu - tous les attributs positifs de Dieu sont essentiellement différents des attributs humains de même nom. Retenons ici pour notre propos le seul attribut "esprit": l'esprit divin en tant qu'attribut de l'Ens a se inclut qu'il doit être compris comme esprit absolu, c'est-à-dire comme esprit se fondant exclusivement en luimême, c'est-à-dire encore comme liberté absolue ou auto-détermination absolue (!) (cfr. GW 5, 186). Nous verrons dans la suite que cette belle théorie de l'esprit divin va connaître dans la suite un "développement considérable", pour employer l'euphémisme schelerien, tout comme va disparaitre sa mise à l'écart radicale d'un "Dieu qui se fait" (GW 5, 219; en français dans le texte).

Enfin quelle est la force qui nous pousse à vouloir connaitre Dieu? Vu de l'autre côté: quelle est la force qui pousse Dieu à vouloir se communiquer? Il faut savoir que pour Scheler l'acte de connaitre tout comme l'acte de vouloir d'ailleurs, n'est pas, comme pour Aristote par exemple, un acte "premier" en ce sens qu'il est fondé dans un acte plus originaire, à savoir l'amour. Nous avons déjà signalé que c'est l'amour infini de Dieu qui le contraint en quelque sorte de se communiquer; de même c'est en dernier lieu l'amour qui incite l'homme à se mettre à la quête de Dieu, à vouloir connaître Dieu (vouloir et connaître étant des actes spirituels fondés dans l'amour, lui-même un acte spirituel. Acte spirituel non rationnel, non théorique appartenant à la sphère de l'émotionnel ou de l'affectif qui possède un pouvoir de connaissance propre, une fonction cognitive propre). À cet égard l'amour schelerien est comparable à l'Eros de Platon, mais chez Scheler il ne peut évidemment pas être réduit à cette fonction instigatrice de la connaissance puisqu'il est principalement amour de soi, amour du prochain et amour de Dieu.

Voilà esquissée en quelques traits la situation d'avant la Kehre. D'un côté un Dieu parfait, tout-puissant, omniscient, amour infini et qui, du fait même de cet amour, a créé le monde, et l'homme à son image; de l'autre l'homme - imparfait sur toutes ses coutures et en pleine dépendance vis-à-vis de son créateur. Pas étonnant donc que le pape Jean-Paul II, Karol Wojtyla, se soit senti attiré (attirance critique évidemment) par ce "moment" de la pensée schelerienne ${ }^{5}$ puisqu'il y va d'une fondation philosophique de la vision chrétienne de la relation entre l'homme et Dieu.

Jusqu'à présent nous n'avons mis l'accent que sur ce qui sépare les deux; en prévision de ce qui va suivre, jetons maintenant un bref coup d'œil sur ce qui les

Cfr notamment WOJTYLA Karol, Primat des Geistes. Philosophische Schriften, Stuttgart, Seewald Verlag, 1979. - The Acting Person, Dordrecht-Boston-London, D. Reidel, 1979. 
unit. Pour cela nous devons quitter la sphère de la connaissance pour entrer dans celle de l'agir. Scheler lui-même nous aide à passer de l'une à l'autre en disant que "si Dieu est pensé comme personne, alors le savoir sur cette personne n'est nullement pensable comme savoir objectif, mais uniquement comme cogitare, velle, amare 'in Deo', c'est-à-dire comme co-effectuation (Mitvollzug) de la vie divine" (GW 7, 220). La valeur d'un acte dépend du fait qu'il est accompli en ou en dehors de Dieu. Scheler ne laisse planer aucun doute à ce sujet: tout comportement de l'homme, pour être bon, doit être un comportement "en" Dieu (amare "in" deo, velle "in" deo, credere "in" deo), c'est-à-dire "un comportement tel que l'acte de la saisie (Einsicht) accompli par l'homme et l'acte de la volonté qui la suit sont vécus et donnés de manière immédiate comme réellement distincts de l'acte divin lui-même, mais comme identiques quant au contenu et comme coïncidant avec le contenu des actes divins de connaissance et de vouloir" (GW 2, 220). Trois remarques nous semblent devoir être formulées concemant ces passages: 1) Durant les années précédant le "toumant", Scheler s'obstine à mettre le in 'deo' entre guillemets, et ce probablement pour éviter tout soupçon de panthéisme. Ils vont disparaître après 1920. 2) A cette époque il limite l'identité de l'acte humain et de l'acte divin au seul contenu de l'acte et insiste sur la différence de la réalité des deux actes. Cette distinction va disparaître également. 3) Il annonce en quelque sorte lui-même l'évolution en parlant de Mitvollzug. Alors qu'avant 1920-1922 cette co-effectuation se situait dans la perspective d'une "imitation" (Nachfolge) de Jésus-Christ, elle prendra une toute autre dimension dans la demière philosophie de Max Scheler.

\section{2}

Et dans cette nouvelle dimension la tâche deviendra plus ardue tant pour l'homme que pour Dieu. Pour bien marquer d'entrée de jeu le tournant - qui d'ailleurs à nos yeux ressemble plus à une révolution qu'à une évolution - nous nous référerons à un des derniers sinon au dernier écrit de Scheler paru quelques jours avant sa mort sous le titre "Philosophische Weltanschauung" où il dit un peu avant la fin: "En son être-homme qui est un être de la décision, l'homme porte la dignité supérieure d'un compagnon d'armes (Mitstreiter), oui d'un coopérateur (Mitwirker) de Dieu; il doit porter au devant de toutes les choses l'étendard de la divinité, l'étendard de la 'Deitas' qui ne se réalise qu'avec le processus du monde, et ce dans la tourmente du monde" (GW 9, 84). Le texte est on ne peut plus clair: d'un co-effectuant des actes divins sub specie imitationis l'homme est passé au stade de véritable coopérateur, d'associé au sens fort. - Avant d'analyser plus avant cette nouvelle collaboration, il est peut-être opportun de dessiner la toile de fond sur laquelle s'est dessiné ce "développement considérable" de sa pensée qui - il le concède lui-même - a subi dans une question aussi fondamentale que la métaphysique de l'être un et absolu (auquel il tient comme auparavant) une modification si profonde qu'il ne peut plus se nommer "théiste" (au sens conventionnel) (cfr GW 2, 17). Est déjà indiqué par là implicitement que ses investigations philosophiques ont changé de cap: alors qu'avant le tournant ses recherches étaient 
centrées sur l'élaboration d'une phénoménologie des valeurs et d'un personnalisme phénoménologique sur lesquels s'est greffée une philosophie de la religion (romaine) - les deux premiers étant maintenus jusqu'au bout et la troisième abandonnée - le centre de gravité de sa pensée sera désormais l'élaboration d'une métaphysique. "Le but suprême de son travail fut la métaphysique. Il a mené les analyses les plus détaillées en sociologie, épistémologie, éthique et psychologie, mais si on lui avait posé la question de ce qui était le plus important pour lui, il aurait certainement répondu: la métaphysique. Le savoir donc sur le fondement universel (Weltgrund), sur l'être absolu, sur la relation dans laquelle se trouvent l'homme, les animaux et toutes les choses avec cet être qui n'est plus contingent comme ceux-là, mais qui est tout simplement absolu et éternel" ${ }^{\prime 6}$. Cette citation provient d'un hommage rendu à Max Scheler à l'occasion de sa mort en 1928 par Max Horkheimer qui, tout comme le fera d'ailleurs Heidegger, avait tenu à intenompre son cours pour faire ce qu'il est convenu d'appeler une laudatio.

Comment notre philosophe conçoit-il ce nouveau centre de gravité? Il en donne deux déterminations qui sont presque autant de définitions puisqu'il y est très explicite: "Ma métaphysique est un panenthéisme personnalistico-idéal de type émanatiste. Émanation: Dieu laissa le monde sortir hors de lui, à travers lui". Et un peu plus loin: "Ma métaphysique est panenthéisme du monde - mais du devenir. Le monde comme histoire est une phase, une destinée, une époque de l'auto-devenir divin - un usinage (Werkgestaltung) dans et à travers lequel le maitre d'œuvre croît et parvient - en le réalisant - à son essence. Dieu dévoile son essence dans l'histoire en se réalisant lui-même" (GW 11, 263-264). D'un côté donc Scheler refuse d'être encore considéré comme théiste, de l'autre, et ce malgré les belles pages qu'il a consacrées à Spinoza (GW 9, 171-182), il repousse la qualification de panthéisme, dont toutes les formes ont en commun l'identité substantielle entre Dieu et le monde; Scheler y oppose une multitude de substances dont la substance divine, multitude qui n'empêche nullement l'immanence absolue de Dieu dans le monde. Il parle dans ce contexte d'une identité réciproque en devenir non seulement de l'homme in Deo, mais aussi de Dieu in hominem et per hominem (cfr. GW 8, 360).

Que cette nouvelle conception bouleverse profondément la relation de l'homme à Dieu et réciproquement est aisé à imaginer. Pour la comprendre véritablement, il nous faut encore signaler le dualisme accentué sinon outré qui caractérise sa dernière philosophie. Dans l'Ens a se il distingue deux attributs, une Pulsion (Drang) toute-puissante et un Esprit (Geist) sans force, sans pouvoir propres. Au point de départ ces deux attributs étaient dans un état de tension originaire et toute l'histoire, tout le devenir consiste à dé-tendre cette opposition originaire par l'idéification de la Pulsion ou, comme il le dit également, par la spiritualisation de la vie. L'espace nous manque ici pour nous étendre sur ce sujet. L'essentiel en est consigné dans "La situation de l'homme dans le monde" de 1927. Retenons seulement quelques conclusions concemant la relation de l'homme à Dieu. "Pour

6 HORKHEIMER Max, Max Scheler (1874-1928), in Gesammelte Schriften Bd. II: Nachgelassene Schriften, Fischer, 1987, p. 147. 
nous le rapport fondamental de l'homme au fondement universel consiste en ce que ce fondement se saisit et se réalise immédiatement dans l'homme qui, en tant que tel, n'est, aussi bien comme être spirituel que comme être vivant à chaque fois qu'un centre partiel de l'Esprit et de la Pulsion de 'l'Étant-par-soi'" (GW 9, 70). Le processus par lequel Dieu se réalise (Gottwerdung) est le même que celui où l'homme se réalise (Menschwerdung) et il consiste en une interpénétration progressive de l'Esprit et de la Pulsion. Ce n'est que dans l'homme que ces deux attributs de l'Ens a se (les deux seuls que nous puissions connaitre) sont rapportés l'un à l'autre de manière vivante. L'homme est leur lieu de rencontre. Dieu et l'homme sont donc à la merci l'un de l'autre. "Aussi peu l'homme peut-il accomplir sa destinée sans se savoir membre de ces deux attributs de l'être suprême, sans savoir qu'il habite en nous, aussi peu l'Ens a se peut-il accomplir sa destinée sans la collaboration de l'homme" (GW 9, 71).

Puisque nous avions privilégié dans la première partie la sphère de la connaissance, commençons, par souci de parallélisme, par examiner cette même problématique dans la nouvelle perspective. Assez curieusement on pourrait prétendre, à première vue, que rien n'a changé: l'homme reste le chercheur de Dieu. Mais dès qu'on y regarde d'un peu plus près, on constate à quel point le changement est profond. Alors que le Gottsucher d'avant 1920-1922 devait pour ainsi dire se contenter de saisir ce que Dieu voulait bien communiquer de lui-même, d'être le réceptacle quasi passif de l'auto-révélation divine, il doit maintenant s'engager dans la même voie - qui mène à Dieu - mais cette fois de manière active et dynamique puisqu'il y est profondément et même essentiellement impliqué. Un aphorisme de Scheler résume à merveille la nouvelle tâche ainsi assignée à l'homme; à la limite on pourrait s'imaginer que Scheler lui aussi se serait senti interpellé par l'inscription delphique qui invitait l'homme à se connaitre soi-même: "Alors que l'homme - se cherchant - se trouve, ... il trouve - 'Dieu'" (GW 9, 296). - Il y a, selon Scheler, une connexion nécessaire et de nature essentielle (wesensnotwendig) entre la conscience de soi et la conscience formelle de Dieu - "formelle" parce que Dieu ne signifie ici que l'être-par-soi-même muni du prédicat sacré, le "contenu" pouvant prendre mille et une configurations. Et cet Ens a se dévoile son être dans l'histoire en se réalisant lui-même. Ce n'est donc pas une substance éternelle qui prend conscience de soi en l'homme et en elle du monde, comme chez Spino$\mathrm{za}$, ni qui prend de plus en plus conscience de soi dans l'histoire du monde, comme chez Hegel, non: l'être suprême n'est connaissable (erkennbar) en l'homme que dans la mesure où celui-ci "réalise dans son centre, dans son cœur pour ainsi dire, la revendication idéale de la déité"(GW 12, 213). L'homme est cet être dans lequel le Ens a se prend conscience de ses deux attributs et de leur tension. C'est en l'homme et à travers l'homme que la déité se réalise elle-même. Il faut donc abandonner la perspective du soumis ou l'image de l'enfant de Dieu pour passer à celle de coopérateur. L'homme co-produit véritablement Dieu et le lieu où se produit cette auto-déification est le cœur humain

Quelle est alors la seule voie qui nous donne un accès cognitif à Dieu? "On peut participer à la vie divine et à son actualité uniquement grâce à la co- 
effectuation, uniquement par l'acte d'engagement et l'acte de l'identification active" (GW 9, 71). Dieu - comme toute- personne, ce qu'il reste chez le dernier Scheler - n'est pas saisissable par des actes "objectivants" (objektivierende), il ne l'est que dans l'acte de l'engagement personnel de l'homme pour la déité. Et Scheler de conclure "La situation de l'homme dans l'univers": "Ce n'est que dans l'engagement de la personne elle-même que s'ouvre la possibilité d'acquérir un 'savoir' sur l'être de l'étant-par-soi" (ibid.). Connaitre Dieu équivaut donc maintenant à saisir son dévoilement progressif dans l'histoire, l'histoire qui est le processus de son auto-réalisation, et cette auto-réalisation à son tour n'est possible que grâce à la participation active de l'homme. - Avant d'examiner d'un peu plus près cette auto-réalisation - conjointe de Dieu et de l'homme dans le monde nous voudrions brièvement évoquer une autre dimension de ce savoir. Dans "Les formes du savoir et la culture" de 1925 (GW 9, 85-119) où il reprend les résultats de recherches antérieures (à partir de 1921 environ) publiées sous le titre "Problèmes d'une sociologie du savoir" (GW 8, 15-190), il assigne au savoir en général trois objectifs suprêmes, et chacun de ces objectifs ne peut être atteint que grâce à sa forme spécifique. In y établit une hiérarchie "claire et immédiatement évidente" entre ces trois formes du savoir qui correpond, selon lui toujours, exactement à la hiérarchie objective des valeurs qu'il avait élaborée dans le Formalisme. Au bas de l'échelle l'objectif est la domination et la transformation pratique du monde à des fins utilitaires; le savoir qui y correspond, Scheler le nomme savoir de domination ou de performance (Herrschafts - oder Leistungswissen). À un niveau supérieur il s'agit de promouvoir le devenir et le plein épanouissement de la "personne qui sait" - c'est le savoir de culture ou de formation (Bildungswissen). Et au troisième et plus haut degré le savoir salvateur ou le savoir rédempteur (Heils- oder Erlösungswissen) qui est un savoir en vue de (umwillen) la déité, c'est-à-dire voué au devenir du monde et au devenir a-temporel de son fondement universel, ces deux devenirs ne pouvant accomplir leur destinée que dans notre savoir humain. Dans ce savoir la personne humaine cherche à prendre part à l'être suprême lui-même ou, vu de l'autre côté, le fondement universel fait offre d'une prise de participation à l'entreprise commune. - Chaque forme de savoir possède ainsi son objectif propre - le "savoir pour le savoir" n'existe pas pour Scheler mais c'est surtout l'objectif "final" de la forme suprême qui lui tient à cœur; à ce niveau l'homme doit être considéré comme co-fondateur, co-formateur et coauteur de la totalité qui se fait, de la déité qui devient et - notre auteur ne cache pas son optimisme - qui se réalisera: "le Dieu qui devient croît dans son œuvre, s'élève et triomphera un jour" (GW 12, 213).

Ce volet rédempteur et libérateur du Heilswissen montre à quel point la "dernière métaphyique" de Max Scheler s'inscrit dans un contexte profondément religieux - l'acte religieux non plus compris comme croyance et imitation qui seraient fondées dans la révélation, la grâce et l'illumination divines, mais comme participation active et spontanée, en pleine "connaissance de cause" commune à Dieu et à l'homme, à la réalisation de la totalité, à ce qu'il appelle l'autodéification. Même si cette dimension religieuse semble ici en quelque sorte privi- 
légiée, elle s'inscrit néanmoins dans une perspective plus large, à savoir la dimension anthropologique, celle de l'homme tout entier.

Sur ce point tous les témoignages, et ils sont nombreux, sont unanimes; ce qui préoccupait avant tout et en premier lieu le Scheler d'après 1920, c'était l'anthropologie métaphysique, cette science fondamentale de l'essence et de la structure essentielle de l'homme, de sa relation à la nature (inorganique), aux plantes et aux animaux et au fondement de toute chose. D'ailleurs il avait demandé peu avant sa mort un semestre sabbatique à l'université de Francfort pour rédiger un ouvrage qu'il avait annoncé depuis quelque temps: son anthropologie philosophique, tout heureux qu'il était - selon ses propres dires quelques semaines avant sa disparition - de constater que la majeure partie des problèmes philosophiques qu'il avait traités jusque là convergeaient dans ce questionnement, questionnement où se rejoignent sinon fusionnent ses aspirations métaphysiques et sa quête sur la situation de l'homme dans l'univers. Ce n'est que dans l'homme et à même l'homme qu'on peut saisir la totalité qui comprend donc, outre l'homme, la nature (inanimée et animée) et Dieu. Le noyau de toute l'histoire est l'histoire de l'homme puisque l'homme est micro-cosme, c'est-à-dire l'unité de toutes les régions eidétiques (Wesensregionen) de l'étant. Micro-cosme certes, mais, dans la nouvelle perspective panenthéiste, également micro-theos (cfr. GW 9,83 ) puisque la personne humaine est "auto-concentration de l'esprit divin luimême" (GW 11, 156) tandis que le monde est le "corps propre" (Leib) de Dieu, à savoir la "corporéisation" (Verleiblichung) continue de la Pulsion éternelle. Le "Dieu qui devient", la déité se réalise donc elle-même selon ses deux attributs essentiels: la Pulsion dans le monde et le corps des êtres humains, l'Esprit exclusivement dans des personnes. C'est donc l'homme en tant que personne qui est le lieu propre de l'auto-déification. C'est en lui que l'évolution universelle, que la déité réalise son être. La déité devait, pour se réaliser, s'accommoder de l'homme; elle ne mérite le qualificatif de Dasein divin que dans la mesure où elle réalise sa déité éternelle dans et par l'homme (cfr GW 9, 55). L'homme - un petit instant de fête dans l'évolution universelle - est par conséquent le moment significatif de la destinée de la déité elle-même. L'histoire de l'homme n'est pas "un simple spectacle pour un observateur et juge divin étemellement parfait, mais est impliquée dans le devenir de la divinité elle-même" (GW 9, 101-102).

Ce bref exposé de la nouvelle perspective nous semble rendre manifeste que ce n'est pas seulement le fond de la pensée schelerienne qui a subi un profond changement, mais également - par nécessité interne probablement - la forme: le langage qui véhicule le panenthéisme se rapproche de plus en plus du langage mystique, s'éloigne de plus en plus du discours phénoménologique. D'ailleurs Scheler lui-même en est parfaitement conscient: d'un côté il fait régulièrement allusion aux mystiques et plus particulièrement à Maitre Eckhart, de l'autre il concède que "la métaphysique partage avec la religion la tentative d'une participation à l'étant absolu, mais non plus comme la religion par croyance ou imitation ... mais par connaissance spontanée ... de la chose ellemême" (GW 8, 87). Cette 
dernière expression tirée du slogan husserlien n'est pas à prendre pour un hasard ou un dérapage isolé; le second Scheler fait encore régulièrement appel à la saisie des essences, à la connexion essentielle, se réfère donc explicitement à la phénoménologie mais à la fin de sa vie (il est impossible de donner une date exacte, mais sûrement après la parution de Sein und Zeit en 1927), il note en bas de page: "Moi, personnellement, j'évite le mot de phénoménologie" (GW 9, 285). Ce qui signifie à nos yeux que Scheler s'est finalement rendu compte d'une inadéquation grandissante entre le langage phénoménologique et sa dernière Sorge, à savoir la destinée de la totalité où sont intimement imbriqués la déité, le monde et l'homme. Et pour expliciter cette imbrication, Scheler utilise un concept qui ne l'a pas quitté sa vie durant, qui n'est ni proprement mystique ni spécifiquement phénoménologique, et dont la signification suit l'évolution générale de sa pensée: le concept de solidarité.

Dans sa période "classique" il énonce son principe de solidarité comme une loi fondamentale d'un cosmos de personnes morales finies, l'énonce même comme l'axiome suprême de toute philosophie sociale et de toute éthique sociale où sont co-responsables toutes les personnes individuelles et les "personnes communes" (Gesamtpersonen), ' des unités sociales d'ordre supérieur à la masse, la société et la communauté vitale). La co-responsabilité est réciproque entre la personne singulière et la personne commune et n'exclut pas leur double auto-responsabilité ... Mais, singulière ou commune, la personne est toujours responsable devant <souligné par nous> la personne des personnes, devant Dieu, et cela tant en ce qui conceme son auto-reponsabilité que sa co-responsabilité (GW 2, 522). Impossible ici d'entrer dans le détail d'une explicitation de ce que Scheler entendait par Gesamtperson, l'important était de souligner l'auto-responsabilité de chaque personne et la coresponsabilité entre les personnes finies devant Dieu. Ce "devant" va disparaître au profit d'un in ou d'un mit. Le principe de solidarité est ainsi reformulé de manière radicale. Sans perdre complètement sa dimension sociale, il devient avant tout métaphysique en ce sens que la co-responsabilité fondamentale est celle entre l'homme et la déité. "Car l'homme est l'exécutant (Vollstrecker) de l'ultime objectif du devenir de la divinité elle-même - en tout cas le co-exécutant - oui, il est l'exécutant qui dirige et qui préside à l'histoire" (GW 12, 223). Ce n'est donc pas "au-dessus" ou "hors" de l'homme que la déité se réalise, c'est dans l'homme qu'elle mène "son dernier combat".

C'en est ainsi terminé avec la quiétude divine - avec un Dieu qui serait autodétermination absolue, toute-puissance sans faille qui aurait créé, dans un geste exubérant d'amour, l'homme, avec un Dieu dont la pré-science n'était que prévoyance ... non nous voilà en présence d'un Dieu qui devait créer ou mieux, qui devait laisser advenir l'homme pour pouvoir se réaliser lui-même. Que les conséquences d'une telle appréhension soient "dramatiques" (au sens noble) pour Dieu également, nul n'en doutera. Scheler lui-même d'ailleurs ne laisse planer aucun

7 Nous reprenons ici la traduction de Maurice de Gandillac à qui nous devons le courage d'avoir transposé en français le Formalisme (paru à Paris chez Gallimard). 
doute à ce sujet: "Je professe le Dieu qui souffre, qui lutte mais qui, en surmontant ses souffrances, est plus heureux (seliger) qu'un Dieu parfait" (GW 11, 263). Ou encore, en réponse à des détracteurs: "On m'a dit que l'homme ne saurait supporter un tel dieu en devenir 'imparfait'; il ne saurait avoir aucune signification pour lui puisqu'il ne pourrait pas 's'appuyer' sur lui, ne saurait être pleinement confiant que la substance éternelle puisse atteindre sa destinée du devenir-dieu et en elle le monde et l'histoire. A cela je réponds que Dieu n'est pas une 'béquille' pour des faibles et que la foi véritable n'est pas une 'compagnie d'assurances', et que cela dépend justement de toi, mon frère ou ma sœur qui parle ainsi, de ton comportement et de ton agir, si la substance devient plus ou moins Dieu ou si lui continue, en toi ou en dehors de toi, de souffrir de sa tension originelle.

Celui qui ne sait supporter un Dieu imparfait, un Dieu qui devient, un Dieu partiellement dépendant de l'action de l'homme, mais uniquement un 'père toutpuissant et infiniment bon qui le protège', celui-là n'est pas encore mûr pour notre époque" (GW 12, 213).

Ce qui, à nos yeux, était devenu insupportable pour Scheler était l'idée qu'un Dieu parfaitement bon (allgütig) ait, suite pour ainsi dire à une "overdose" d'amour, créé le monde et l'homme et reste dans la suite insensible au destin de son œuvre, reste indifférent à ce qui se passe au Kosovo, en Colombie, au Cambodge ou en Éthiopie - pour ne citer que quelques exemples actuels, Scheler faisant allusion au tremblement de terre au Japon et à la première guerre mondiale. Pour notre philosophe, la perfection n'est pas au début mais n'est que l'aboutissement d'une longue marche où cheminent main dans la main Dieu et l'homme.

Que ce "développement considérable" de la pensée de Max Scheler que nous venons d'exposer ait suscité de nombreux commentaires, réactions et interprétations n'étonnera personne. La plupart toument évidemment autour de la question du pourquoi de cette évolution/rupture. Il va de soi que nous ne pouvons en faire ici un relevé exhaustif. Signalons donc simplement leur existence tout comme la pantion d'une excellente étude relativement récente sur ce sujet de la main de Wemer Schüßler. ${ }^{8}$ Tout en faisant le point sur la question il propose une thèse nouvelle: que l'argument décisif pour l'idée d'un "Dieu qui devient" serait issu du problème du mal. Personnellement nous pensons que cette nouvelle piste devrait être exploitée mais surtout élargie en direction du "principe" de la responsabilité ou mieux, dans le cadre de la dernière philosophie de Scheler, de la co-responsabilité. Mais ceci devrait être l'objet d'une étude à part.

Ce qui nous semble indiscutable, c'est le fait que Scheler était à la recherche d'une voie moyenne entre l'affirmation "L'homme est la mesure de toutes choses" de Protagoras et le "C'est donc Dieu qui serait pour nous au plus haut degré la mesure de toutes choses, et Lui bien plutôt, je suppose, que ne l'est, au dire de certa-

8 SCHÜSSLER Werner, "Solidarisch mit der Gottheit". Mensch, werdender Gott und das Problem des Ubels beim späten Scheler, in Phänomenologische Forschungen (Neue Folge 3, 1998 - 2. Halbband), pp. 159-179. 
ins, tel ou tel homme" de Platon (Les Lois IV, 716). À la recherche donc d'un Dieu à notre portée, c'est-à-dire qui nous soit accessible ou, autrement dit encore, que nous puissions comprendre. N'est-ce pas pour cette raison qu'il a voulu en quelque sorte "ramener Dieu sur terre"? Peut-on, doit-on lui adresser pour cela le reproche, sinon lui attribuer la faute d'hybris, cet orgeuil démesuré qui pousse l'homme à vouloir être l'égal de Dieu? Nous sommes convaincus que ce n'était pas l'enjeu de la Kehre schelerienne. Vers la fin de sa vie il écrit: "Jusqu'à maintenant l'homme a pensé trop petit de lui-même - et non trop grand de la divinité" (GW 11, 263). Ce rehaussement de l'homme, en le rendant responsable de la destinée de la déité, ouvre l'horizon d'une co-responsabilité où il ne saurait plus être question de se rejeter la faute mutuellement. L'homme, l'humanité doit assumer ! Encore une fois une voie moyenne entre deux positions extrêmes: déclarer ou bien Dieu seul responsable de tout y compris le mal ou bien l'homme. Une autre possibilité serait évidemment mais elle n'aurait pu être envisagée par Scheler - d'éliminer carrément Dieu.

Le discours philosophique ne nous permet pas d'aller plus loin dans ce questionnement. Nous avons voulu montrer jusqu'où il peut aller - sans transgresser les limites, à nos yeux interdites pour la seule raison qui le sépare du verbe des théologiens. - Si nous sommes ainsi convaincus que notre propos a respecté ces limites et est resté philosophique, nous voudrions maintenant aborder une question plus délicate, un problème plus difficile à résoudre: notre discours est-il resté phénoménologique? La même question formulée de manière plus générale: quelles sont les limites de la démarche phénoménologique? La fondation d'une nouvelle société de phénoménologie n'est-elle pas un moment privilégié pour s'interroger sur l'essencemême de la phénoménologie, sur ses possibilités et ses limites?

Afin d'ouvrir le chemin de la réponse, nous proposons de rappeler d'abord le § 24 des Ideen I qui constitue en quelque sorte la charte du mouvement phénoménologique: "Avec le principe des principes nulle théorie imaginable ne peut nous induire en erreur: à savoir que toute intuition donatrice originaire est une source de droit pour la connaissance; tout ce qui s'offre à nous dans l'intuition', de façon originaire (en chair et en os pour ainsi dire) doit être simplement reçu pour ce qu'il se donne, mais sans non plus outrepasser les limites dans lesquelles il se donne alors". Sommes-nous astreints à nous tenir à cette "charte" sous peine d'exclusion du mouvement? Ou, pour pousser plus loin notre questionnement: sommes-nous obligés d'adhérer à cet idéal husserlien d'une phénoménologie comme science rigoureuse et en même temps comme philosophie première qui l'a conduit, pour qu'elle reste "pure", à une philosophie de la constitution et à l'idéalisme transcendental? Un bref coup d'œil sur la suite du mouvement phénoménologique suffit pour répondre par la négative. Mais ceci ne résout pas encore notre problème de savoir si le phénoménologue peut aborder - au nom de la phénoménologie - des questions comme celles abordées par le dernier Scheler et dont celui-ci dit lui-même qu'elles se situent au niveau métaphysique. Si on avait soumis au dernier Heidegger la question schelerienne de Dieu comme être un et absolu, celui-ci, s'il avait accepté de répondre, aurait plus que probablement résumé sa propre position en nous lançant: Gott gottet. I n'est pas sûr qu'une telle phénoménologie "tautologique" (die Welt weltet, das Sein west etc.) nous soit d'un grand secours. 
D'ailleurs il va de soi que notre intention se limite à soulever la question et nullement d'y apporter une réponse quelque peu satisfaisante. En France cette question a donné lieu à une polémique dont rendent compte les belles pages de Dominique Janicaud à ce sujet (surtout: Le toumant théologique de la phénoménologie française, Combas, éditions de l'éclat, 1991 et La phénoménologie éclatée, Paris, éditions de l'éclat, 1998). Et, pour en terminer avec cette Fragestellung, peut-être une dernière suggestion inspirée de Max Scheler: ne pourrait-on pas déplacer à nouveau ce débat vers le lieu par excellence où se rejoignent toutes les questions philosophiques: le cœur humain? 